\title{
Spatio-temporal evolution of uranium emission in laser-produced plasmas
}

\author{
S. S. Harilal ${ }^{11}$, P. K. Diwakar ${ }^{2}$, N. L. LaHaye ${ }^{1}$, M. C. Phillips ${ }^{1}$ \\ ${ }^{\text {I}}$ Pacific Northwest National Laboratory, P.O. Box 999, Richland, Washington 99352, USA \\ ${ }^{2}$ School of Nuclear Engineering, Purdue University, West Lafayette, IN 47907, USA
}

Laser-induced plasma spectroscopy provides much impetus as a nuclear forensics tool because of its capability of standoff detection and real-time analysis. However, special nuclear materials like U, Pu etc. provide very crowded spectra, and when combined with shifts and broadening of spectral lines caused by ambient atmospheric operation, generate a complex plasma spectroscopy system. We explored the spatiotemporal evolution of excited $\mathrm{U}$ species in a laser ablation plume under various ambient pressure conditions. Plasmas were generated using $1064 \mathrm{~nm}, 6 \mathrm{~ns}$ pulses from a Nd:YAG laser on a U containing glass matrix target. The role of air ambient pressure on $U$ line intensities, signal to background ratios and linewidths were investigated. Spatially and temporally resolved optical time-of-flight emission spectroscopy of excited uranium atoms were used for studying the expansion hydrodynamics and the persistence of $U$ species in the plume. Our results showed that $U$ emission linewidths increased with pressure due to increased Stark broadening; however, the broadening was less than for Ca. A comparison with $\mathrm{U}$ emission features in the presence of an inert gas showed the persistence of $U$ species in plasmas in ambient air is significantly reduced; this could be due to oxide and other reactive species formation.

Keywords: U emission, Plasma spectroscopy, Laser produced plasma, LIBS, Ambient gas effects, Line broadening, Nuclear forensics

${ }^{1}$ Corresponding author, Email address: hari@pnnl.gov 


\section{Introduction}

Nuclear forensics is a key element of nuclear and national security. Typical nuclear forensics analysis includes the characterization of material and correlation with production history based on isotopic composition of the material (U, Pu etc.) and accompanying impurity elements (typically low-Z materials) [1]. In addition to monitoring special nuclear materials (SNM), there is also a growing interest in realtime monitoring of elements with lighter mass (low-Z) for various applications associated with nuclear forensics. Laser ablation (LA)-based techniques for nuclear forensics provide several key advantages which include little to no sample preparation, applicability to a wide range of materials, no requirement of specific sample dimensions, real-time capability, ultra-trace detection and feasibility of remote analysis. Among the various LA-hyphenated techniques, LA-Inductively Coupled Plasma - Mass Spectrometry (LA-ICP-MS) is routinely used for SNM elemental and isotopic analysis [2, 3]. LA-Laser Absorption Spectroscopy (LA-LAS) is an extremely sensitive method capable of probing elemental as well as isotopic ground and excited state populations [4, 5]. LA - Optical Emission Spectroscopy (LA-OES), commonly called Laser Induced Plasma Spectroscopy (LIPS) or Laser Induced Breakdown Spectroscopy (LIBS), provides another dimension to LA-hyphenated techniques in nuclear forensics where light emission from the plasma plume is analyzed [6-14].

Advantages of LIBS compared to other LA based techniques for nuclear forensics include standoff (non-contact) detection, simultaneous multi-element detection, and real-time analysis. For nuclear applications, the LIBS technique was successfully used for in-situ remote measurements of copper and chromium content in mild steel economizer tubes in nuclear power stations $[15,16]$. Whitehouse et al. [16] reported a novel design for a $75 \mathrm{~m}$ long umbilical fiber-optic LIBS system suitable for remotely determining the copper content of $316 \mathrm{H}$ austenitic stainless steel super-heater bifurcation tubing within the pressure vessels of advanced gas cooled reactor nuclear power stations. The LIBS method has been used for the compositional analysis of radioactive contamination within a hot-cell by transmitting the laser beam through a $1 \mathrm{~m}$ lead-glass shield window [15]. Wachter and Cremers [17] used LIBS to determine $\mathrm{U}$ in solution for possible applications in process control in nuclear fuel reprocessing facilities. 
They reported a detection limit of $100 \mathrm{ppm}$ (4M nitric acid/aqueous) for U [17]. Very recently, LIBS has been successfully developed to quantify thorium (Th) and uranium (U) in a solution using membranebased filter paper as a sample support [18, 19]. However, further research efforts are necessary to extend LIBS capability to use the technique as a reliable nuclear material sensor especially at atmospheric conditions. In addition to measurements of elemental abundance, LIBS has also been used for isotopic characterization of $U$ adding to its value for nuclear forensics applications [6, 20].

LIBS is routinely used for elemental analysis of light elements, but the heavy and electron-rich elements of interest in SNM materials such as $\mathrm{U}, \mathrm{Pu}$, Th, etc. emit crowded spectral features immersed in strong continuum emission (due to free-free and free-bound transitions) [8]. Thus there is a need to characterize and optimize the signal to background (S/B) ratios of elemental lines to improve the analytical merits. The S/B ratios and persistence of emission from species in a laser ablation plume are determined by the temporally- and spatially-dependent physical properties of the plasma including atomic number densities and excitation temperature, which in turn strongly depend on various external factors including ablation laser parameters (wavelength, energy etc.) and environmental factors (nature and pressure of the ambient gas). Recently LaHaye et al. [13] reported the emission properties and persistence of $\mathrm{U}$ in ambient Ar environment. However, laser ablation plume expansion into inert ambient conditions is less complex compared to their expansion in the presence of a reactive gas like air. In the latter scenario, the plasma chemistry will redefine the plume emission features, S/B ratios and persistence of all species in the plume. In this paper, we present time- and space-resolved measurements of uranium emission from a U-containing multi-component plasma to investigate the role of ambient air pressure on $\mathrm{U}$ emission features, S/B ratio, linewidths and persistence of emission. A comparison of $\mathrm{U}$ emission features in Ar and Air ambient environments is also discussed.

\section{Experimental set up}

For laser ablation, $6 \mathrm{~ns}, 1064 \mathrm{~nm}$ pulses from a Nd:YAG laser were used. The laser beam was focused down using a $40 \mathrm{~cm}$ focal length plano-convex lens and the laser spot diameter at the target was 
maintained at $\sim 1 \mathrm{~mm}$. The target used was a glass sample containing $1.3 \% \mathrm{UO}_{2}$. The target $(3 \mathrm{~mm}$ thickness and $25 \mathrm{~mm}$ diameter) was mounted on a motorized translation stage which was moved to avoid target cratering effects. Experiments were performed at varying levels of background air pressure. The emission from the plasma was collected and imaged onto the slit of a $0.5 \mathrm{~m}$ spectrograph using an optical system. The spectrograph was equipped with 3 dispersion gratings and contained two detector exits. The dispersed light from the spectrograph was diverted either to an Intensified CCD camera (ICCD) for the detection of a range of wavelengths or to a photomultiplier tube (PMT) for optical time-of-flight (OTOF) detection of emission from a particular spectral line in the plume. All experiments were performed in a vacuum chamber, capable of pressure varying between atmospheric pressure and $10^{-6}$ Torr. The measurements were carried out at a laser intensity of $\sim 2 \mathrm{GWcm}^{-2}$ (laser fluence $\sim 14 \mathrm{~J} / \mathrm{cm}^{2}$ ) with varying air pressures.

\section{Results and discussion}

Spatially-resolved spectroscopic analysis was carried out on U-containing plasma; a typical spectrum in the wavelength range $350-370 \mathrm{~nm}$ is given in Figure 1 . The spectrum was recorded using $1800 \mathrm{~g} / \mathrm{mm}$ grating with an insertion delay of $1 \mu \mathrm{s}$ after the onset of plasma formation and an integration time of $5 \mu \mathrm{s}$, $2 \mathrm{~mm}$ from the target surface with an air pressure of 100 Torr. The spectral window given in the Figure 1 shows the emission lines mostly from both U I and U II transitions along with strong background emission. The U spectral reference data [21-25] showed nearly 170 U I and U II lines in this spectral region $(350-370 \mathrm{~nm})$, and the most prominent resolved lines are marked in the figure. $U$ is a line-rich element and it is obvious that the database used for line identification is far from complete, although it provided details about the most intense lines. Among the lines identified, U I emitting at $356.18 \mathrm{~nm}$ is well-resolved; we used this excited emission line for S/B ratio estimation as well as for TOF emission analysis.

Figure 1 also shows the presence of strong background emission in the given spectral range. The spectral information given in Figure 1 was collected at an air pressure 100 Torr. At these pressure 
levels, the background gas will confine the plasma to a smaller volume compared to plasma free expansion in vacuum or low background pressures [26] which enhances collisional excitation, leading to increasing line emission along with background continuum. According to Chinni et al.,[7] the presence of strong background emission seen in $\mathrm{U}$ plasmas could be due to overlapping of high density $\mathrm{U}$ lines. However, the target used in the present study is a mass limited $U$ target (1.3\%) and hence further studies are necessary to confirm this.

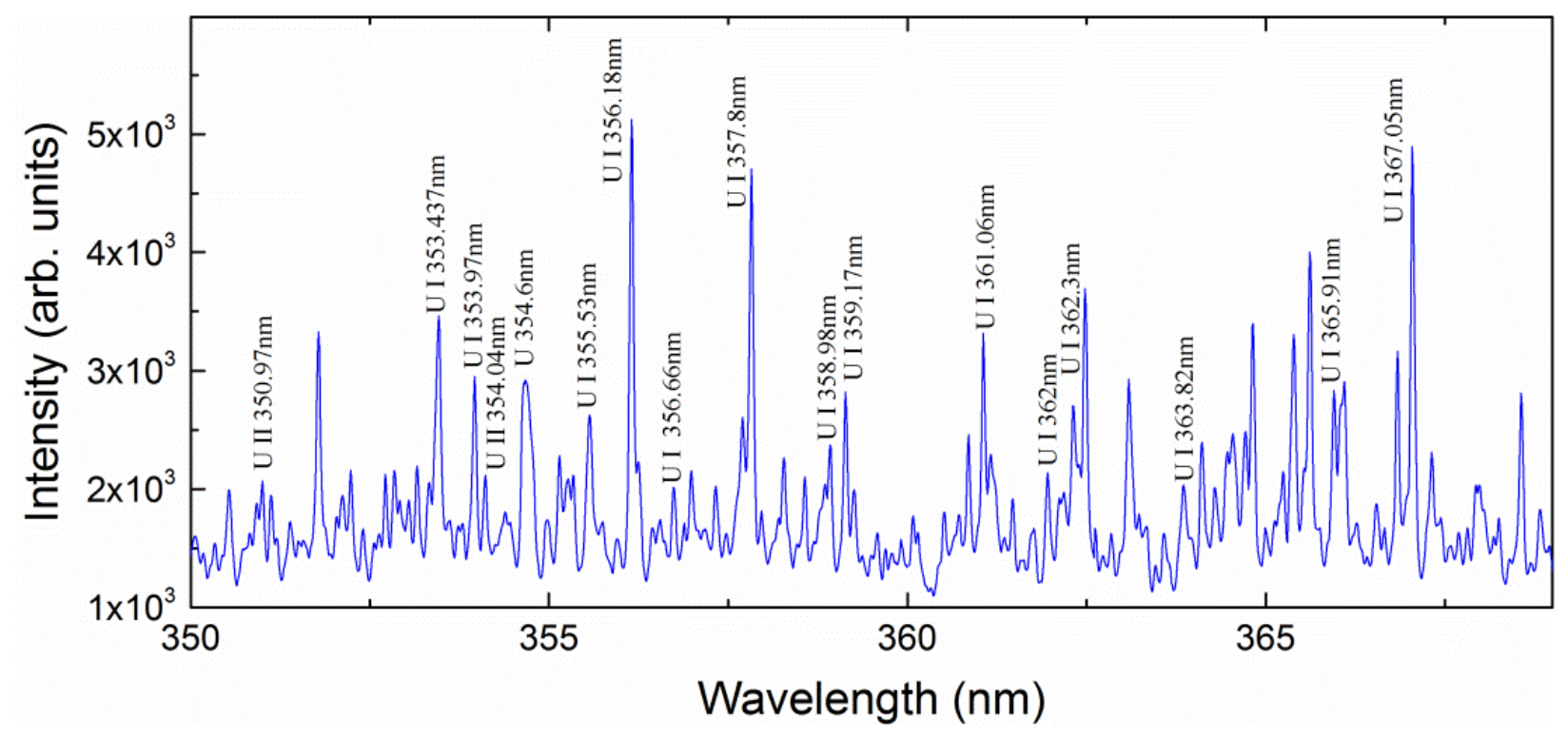

Fig. 1. Typical spectrum of U-containing plasma in the range $350-370 \mathrm{~nm}$ is given. The major $\mathrm{U}$ emission lines are identified. The spectrum was recorded with a delay of $1 \mu \mathrm{s}$ after the onset of plasma formation and with an integration time of $5 \mu \mathrm{s}, 2 \mathrm{~mm}$ from the target surface with a pressure of 100 Torr.

The intensity of line radiation is an important consideration for all emission-based analytical applications. We evaluated the intensity changes of the U I transition emitting at $356.18 \mathrm{~nm}$ with respect to air pressure and results are given in Figure 2. The intensities given are background subtracted, and measurements were made at a distance $2 \mathrm{~mm}$ from the target surface. The recorded spectral intensity is found to increase significantly with the addition of background pressure especially $>1$ Torr pressure levels and reaches a maximum at $\sim 200$ Torr, then drops. Assuming local thermodynamic equilibrium (LTE) exists within the plasma, the intensity of line radiation $\mathrm{I}$ for a transition from $\mathrm{m}^{\text {th }}$ to $\mathrm{n}^{\text {th }}$ level is given by [27] 


$$
I \approx A_{n m} N g_{m} \frac{h c}{\lambda_{m n}} e^{-E_{m} / k_{B} T}
$$

where $A_{n m}$, the transition probability; $N$, the number density; $g_{m}$, the statistical weight; $\lambda_{\text {mn }}$ the wavelength of line emission; $\mathrm{E}_{\mathrm{m}}$, the upper energy of the transition; $\mathrm{k}_{\mathrm{B}}$, Boltzmann's constant; h, Planck's constant; c, velocity of light; and $\mathrm{T}$, the excitation temperature. Thus the emission intensity for a particular spectral line depends on both the number density of the emitting species and on the temperature, both of which vary with time and space in the plasma. Previous reports showed that the addition of a background gas increases both the temperature and density of the plasma due to plasma confinement which eventually affects the population of all excited species in the plume [28]. At lower pressures $(\leq 1$ Torr) the intensity increase with increased pressure is not drastic, indicating that the plumeambient collisional excitation is minimal at these pressure ranges. Along with the increase in line intensity, the background noise level also increased due to a significant rise in temperature caused by the confinement from the ambient gas [28]. Hence it is appropriate to estimate the $\mathrm{S} / \mathrm{B}$ ratio $(\mathrm{S} / \mathrm{B}=$ $\left.\frac{I_{\text {Max }}-I_{\text {Background }}}{I_{\text {Background }}}\right)$, an important consideration for analytical applications. These ratios are calculated from the peak line intensity, $I_{\operatorname{Max}}$, and the averaged background intensity near to the line. It has to be pointed out that both signal to noise $(\mathrm{S} / \mathrm{N})$ and $\mathrm{S} / \mathrm{B}$ ratios are routinely used for comparing performance metrics of emission analysis. Typically $\mathrm{S} / \mathrm{N}$ and $\mathrm{S} / \mathrm{B}$ trends are often similar, but can occasionally be inconsistent due to the transient nature of a signal $[29,30]$.

The variation in S/B ratio with respect to air pressure obtained from $\mathrm{U}$ line emission is also given in Figure 2. Even though more than one order improvement in U signal intensity is observed with the addition of ambient gas pressure, such an improvement is found to be absent when considering the S/B ratio. The S/B ratio showed a more or less constant value $(\sim 2)$ regardless of ambient air pressure levels used. The lack of improvement in S/B ratio could be partly related to the significant rise in background continuum at higher pressures. However, our recent results of $\mathrm{U}$ line emission S/B ratios in Ar ambient showed a factor of $4 \times$ increase with the addition of Ar ambient gas [13]. This indicates that the nature of 
the ambient gas plays a major role in the plasma emission properties. Argon, being an inert gas, promotes collisional excitation through plasma confinement with minimal effects on plasma chemistry. Modeling studies also showed the plasma generated in Ar ambient is typically hotter [31]. Instead, along with collisional excitation of the plume, ambient air also affects the plasma chemistry through oxide and other reactive species formation [32].

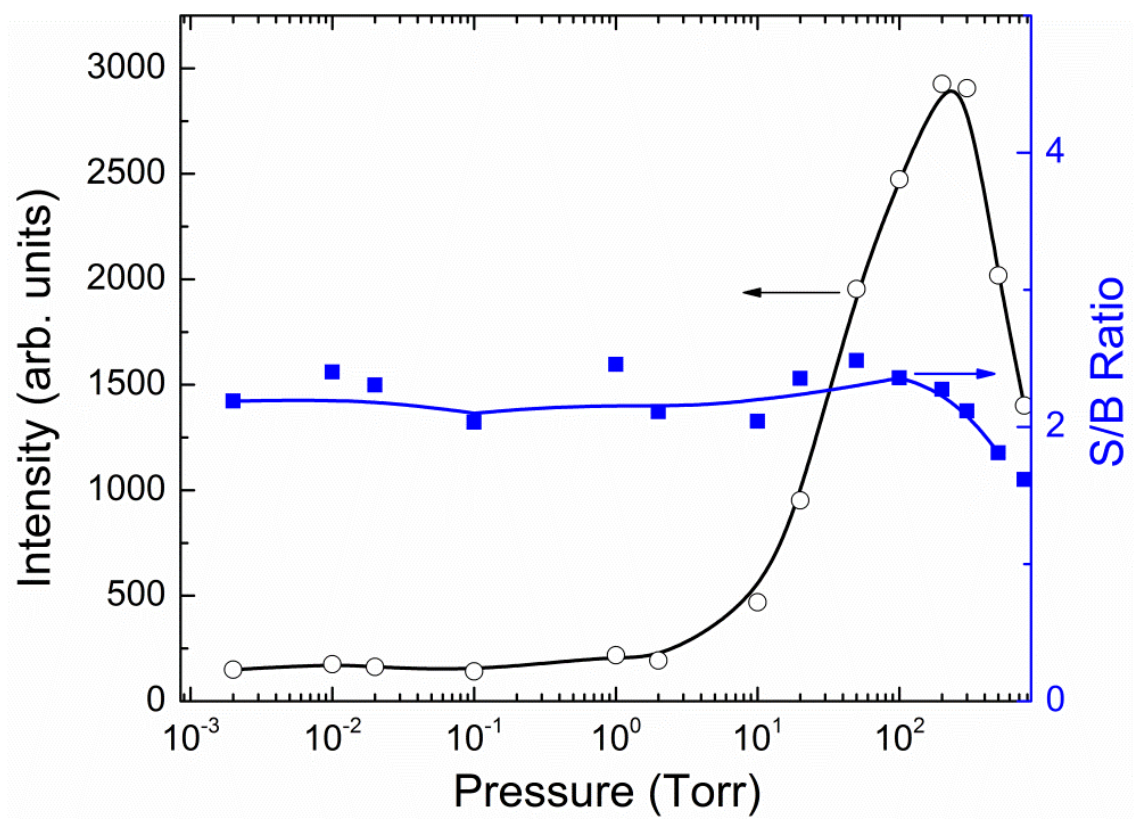

Fig. 2. Intensity and $\mathrm{S} / \mathrm{B}$ ratio of $\mathrm{U}$ line emission at $356.18 \mathrm{~nm}$ is given with respect to air ambient pressure. The measurements were taken $2 \mathrm{~mm}$ from the target with a laser intensity of $2 \mathrm{GW} / \mathrm{cm}^{2}$.

We observed a constant S/B ratio for $\mathrm{U}$ line emission at various pressure levels. Apart from the role of ambient air on plasma chemistry and the background level, the spatial observation position can influence the estimation of absolute line intensities and S/B ratios. Other factors affecting the S/B ratio estimate include the line selection (intensity and hence transition probability, and spectral location), gating time, and gate delay. The fundamental parameters (density and temperature) of laser ablation plumes are highly dynamic even in vacuum, where the expansion is isentropic and adiabatic. The expansion dynamics become much more complex in the presence of an ambient gas [33]. Typically, laser plasmas are hotter and denser at closer distances to the target and at early times, and both temperature and density decay rapidly with time and space. For the laser intensities used in the present study $\left(2 \mathrm{GW} / \mathrm{cm}^{2}\right)$, the density at early times can approach the critical density of the laser beam $\left(1 \times 10^{21} \mathrm{~cm}^{-3}\right)$ and the 
temperature can reach $>10 \mathrm{eV}[34,35]$. According to Singh et al. [36], assuming adiabatic and spherical expansion of the plume, both these parameters decay by $\sim 1 / \mathrm{r}^{3}$ with space where $\mathrm{r}$ is the radius of the plume. However, the presence of an ambient environment with various gas pressure levels will affect the expansion and confinement dynamics and therefore influence the emission features of the plasma [28]. Accordingly, we measured the spatial dependence of S/B ratios at various pressure levels and results are given in Figure 3.

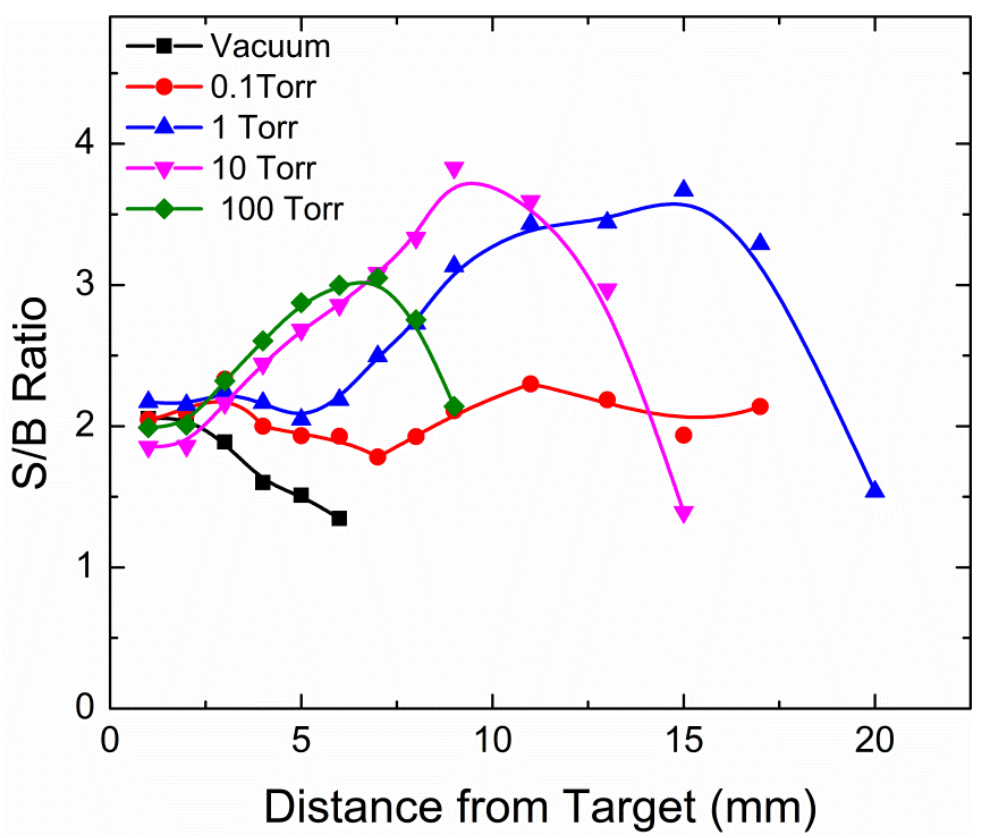

Fig. 3. Spatial dependence of S/B ratio of $\mathrm{U}$ line emission at $356.18 \mathrm{~nm}$ is given for various ambient pressure levels.

The spatial dependence of S/B ratios of U line emission at $356.18 \mathrm{~nm}$ showed different trends at various pressure levels (see Figure 3). The S/B ratios showed a constant value $\sim 2$ for all pressure levels at closer distances $(\leq 3 \mathrm{~mm})$ from the target surface. At vacuum pressure levels, the maximum line intensity and greatest $\mathrm{S} / \mathrm{B}$ ratios are observed closer to the target. At $\sim 100$ mTorr, which is close to the transition regime from a collision-less to collisional system (between plume and ambient species) [26], the S/B ratio is found to be constant regardless of the distance from the target. In this pressure regime the diffusion of ambient gas species into the plume is prevalent. At higher pressures ( 1-10 Torr), collisional excitation is dominant at farther distances, indicated by a rise in S/B ratio. Within this pressure range, the plume- 
ambient boundaries are represented by strong shockwave formation, and collisional excitation leads to intense emission at farther distances from the target [26]. At higher pressure levels $(\sim 100$ Torr $)$, the optimal emission region is moved towards the target because of ambient confinement of the plasma. The spatial dependence of S/B ratios show that the analytical metrics of LA plumes change significantly with space and that the optimal spatial positions for collection vary with ambient pressure.

The width of the spectral emission lines in laser-induced plasmas are broadened by collisional processes perturbing the emitting atoms and ions; thus, the pressure of an ambient gas affects the width of emission lines greatly [4]. The instrumental broadening also must be considered, since this places a lower limit on the width of a spectral line that can be measured. The natural linewidth of a transition is typically small relative to other broadening mechanisms. In the LIP, both Doppler and collisional broadening can be important depending on the ambient pressure. Doppler broadening increases with temperature, and can therefore change with ambient pressure through changes in kinetic temperature of species in the plasma. Typically the dominant line broadening mechanism in laser induced plasmas is Stark broadening due to collisions with free electrons. At later times in the plasma evolution when the electron density has decreased, van der Waals broadening due to collisions with neutral ambient gas species may become dominant; however, this is not typically observed in LIP due to corresponding low emission intensity at these times. Recent high-resolution LA-LAS measurements of the U I $860.796 \mathrm{~nm}$ transition showed a full width half maximum (FWHM) of $0.005 \mathrm{~nm}(2.2 \mathrm{GHz})$ in air atmospheric pressure, at a delay of $7 \mu \mathrm{s}$ after plasma formation [4]. In this case, the linewidth was determined by contributions from both Doppler broadening and collisional broadening with neutral molecules from the ambient air (primarily nitrogen). In addition, a nearly linear dependence of U I absorption linewidth on pressure was measured.

It is important to note that spectral line broadening in a laser induced plasma is both spatially- and temporally-dependent due to variations in temperature and electron density. Typically all emission lines show a maximum width closer to the target as well as at early times where the Stark effect is predominant. Recently Choi et al. [8] evaluated the FWHM of U I and U II transitions with time and found that adequate isotopic separation is possible at later times to determine isotopic ratios. Their time 
resolved linewidth measurements showed that the selection of the detector gate width affects the measured linewidth greatly at early times of plasma evolution. The FWHM of the UI line at $356.18 \mathrm{~nm}$ is given in Figure 4 for various air pressure levels along with the width of the Ca I emission line at 422.67 $\mathrm{nm}$ measured under the same conditions. The FWHM values given in Figure 4 are not corrected for instrumental broadening which is estimated to be $\sim 0.03 \mathrm{~nm}$ using a He-Ne laser. Hence the measured linewidths at lower pressure levels could be partly due to the instrumental broadening limit. Considering the measurements were carried out at a distance $2 \mathrm{~mm}$ from the target surface with an integration time of $2 \mu$ s and with a delay of $100 \mathrm{~ns}$, the Stark effect is expected to be the major broadening mechanism. Doppler broadening for these $\mathrm{U}$ and $\mathrm{Ca}$ transitions at a temperature of $10000 \mathrm{~K}$ is calculated to be 0.002 $\mathrm{nm}$ and $0.005 \mathrm{~nm}$, respectively, which is much smaller than the measured widths even at low pressure and can be neglected.

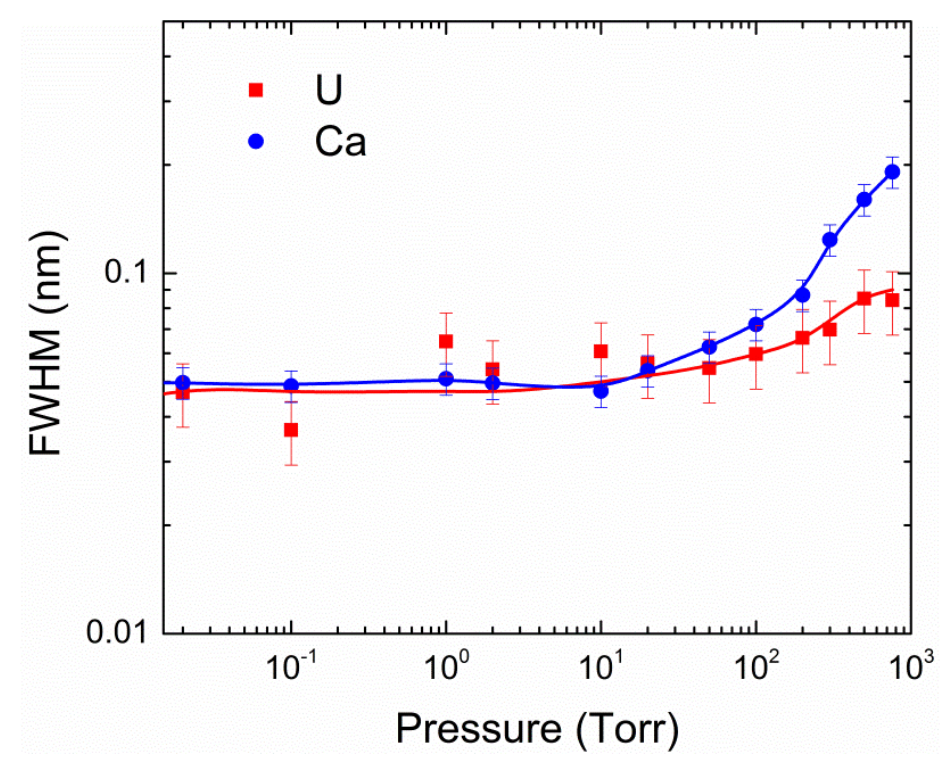

Fig. 4. The estimated FWHMs of U I (356. $18 \mathrm{~nm})$ and Ca I $(422.67 \mathrm{~nm})$ lines are given with respect to various ambient pressure levels.

According to Figure 4, the U I transition linewidth is found to be less influenced by the ambient pressure than the Ca I transition. For example, the recorded FWHM of the U I line is $\sim 0.05 \mathrm{~nm}$ at low pressures (0.1-1 Torr) and increases to $\sim 0.09 \mathrm{~nm}$ when the ambient pressure increased to atmospheric conditions. In contrast, the Ca I transition linewidth is similar to the $\mathrm{U}$ I linewidth at low pressures, but 
increases to $0.2 \mathrm{~nm}$ at atmospheric pressure. The $\mathrm{Ca}$ I transition at $422.67 \mathrm{~nm}$ has similar energy levels and transition probability compared to the $\mathrm{U}$ I transition at $356.18 \mathrm{~nm}$ (transition $0-28067 \mathrm{~cm}^{-1}, \log (\mathrm{gf})=$ 0.3). The large integration time for the measurement results in significant averaging of the rapidly changing electron density and therefore Stark broadening. Comparing the FWHMs of U I and Ca I emission lines obtained at various pressure levels indicates that the U I transition has a lower Stark broadening coefficient, but further study is needed to accurately determine the Stark broadening coefficient for the U I transition.

Since LIPs are highly dynamical systems and their emission features change rapidly with both space and time, spatio-temporal contour mapping of various emitting species in the plume is useful for understanding the plume and species kinetics. OTOF measurements are capable of providing high temporal resolution (as low as $1 \mathrm{~ns}$ ) and are useful in generating fast contour mapping of spatio-temporal evolution of various species in the plume [37, 38]. We employed OTOF to record time-resolved emission from the U I $356.18 \mathrm{~nm}$ line at various locations in the plasma with high spatial resolutions (governed by the slit width of the spectrograph) and used the measured data to generate spatio-temporal contours of $U$ species in the plume at various pressure levels; results are given in Figures 5 and 6 . The contour plots clearly show the role of ambient pressure in extending the U emission both spatially and temporally. At low pressure (eg. 50 mTorr), most of the emission occurred very close to the target and the plasma lifetime is limited to $<500 \mathrm{~ns}$. With increasing pressure, the spatial and temporal extension of U emission are significantly increased. For eg. at $\sim 10$ Torr pressure levels, the $\mathrm{U}$ emission spatial and temporal extensions are increased significantly $(\sim 10 \mathrm{~mm}, \sim 3 \mu$ s respectively). At higher pressures (Figure 6$)$, the spatial extent is reduced compared to that indicated by Figure 5; however, the persistence of emission is longer, as discussed further below. 

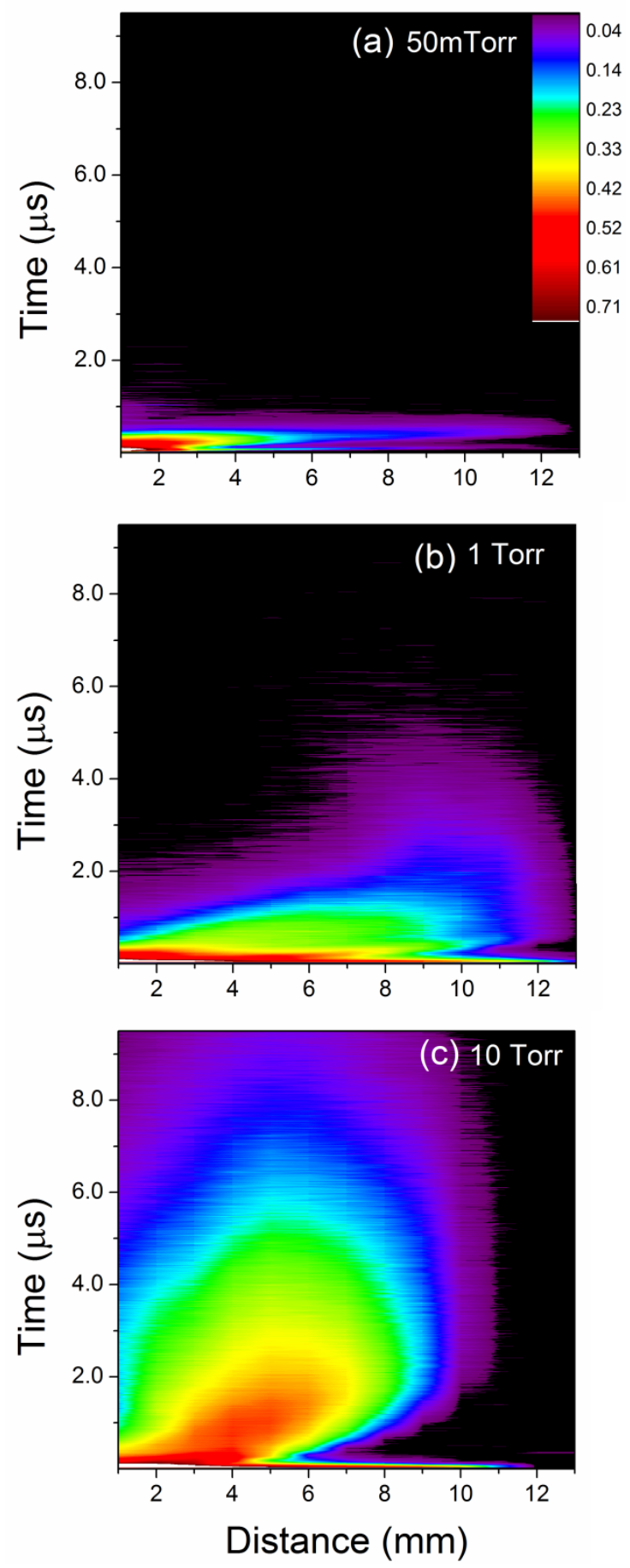

Fig. 5. The position-time contours obtained from U I $356.18 \mathrm{~nm}$ TOF are given for (a) 50mTorr, (b) 1 Torr and (c) 10 Torr air pressure levels. The scale bars given in the left figure correspond to signal levels measured using a PMT and intensity levels are normalized to similar scale levels for all contours. 

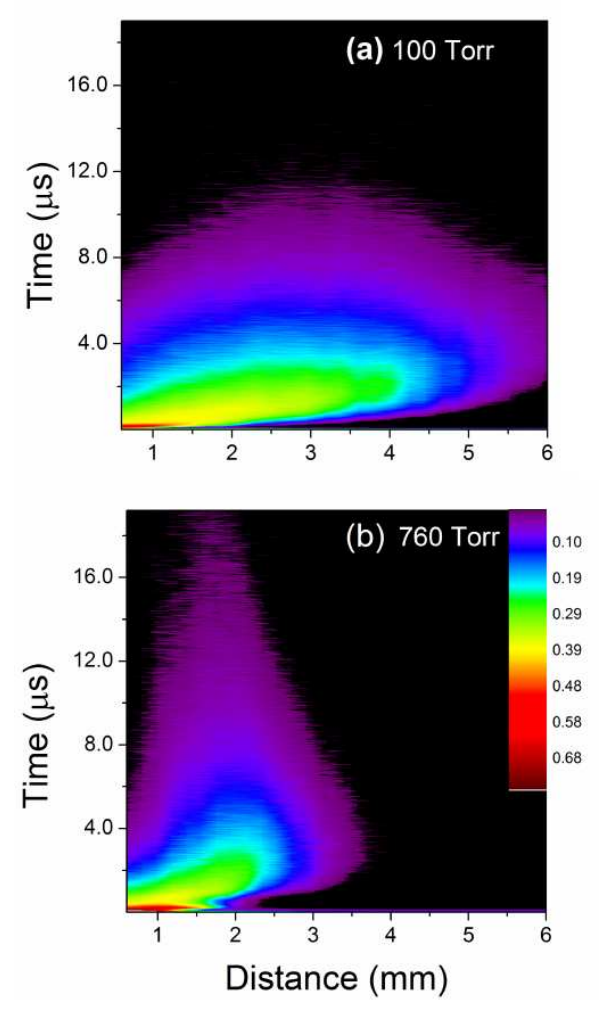

Fig. 6. The position-time contours obtained from U I $356.18 \mathrm{~nm}$ TOF are given for (a) 100mTorr, and (b) 760 Torr air pressure levels. The scale bars given in the left figure correspond to signal levels measured using a PMT and intensity levels are normalized to similar scale levels for both contours.

Individual OTOF profiles of U I emission at $356.18 \mathrm{~nm}$ are given in Figure 7 recorded at $4 \mathrm{~mm}$ from the target surface for $50 \mathrm{mTorr}$ and 400 Torr. Figure 7 shows that $\mathrm{U}$ has a single-peak structure in its temporal profile, preceded by a prompt peak at an earlier time. The prompt peak seen in Figure 7 could be attributed to continuum in vacuum conditions and a combination of continuum and fast electron excited ambient plasma at ambient pressure conditions [39] The temporal profiles of $U$ emission are found to be very sensitive to the pressure of the background gas. The expansion velocity of $U$ species $\left(\mathrm{v} \sim 1.64 \times 10^{6}\right.$ $\mathrm{cm} / \mathrm{s})$ measured at $50 \mathrm{mTorr}$ is reduced to lower values $\left(\mathrm{v} \sim 0.42 \times 10^{6} \mathrm{~cm} / \mathrm{s}\right)$ in the presence of 400 Torr air ambient, while the decay time of the emission increased considerably at the higher pressure. The deceleration of $\mathrm{U}$ species is presumed to be caused by enhanced collisions with the ambient gas species which eventually lead to slower temporal decay for all species in the plume. 
We define plasma persistence or decay time when the peak intensity falls to $1 / \mathrm{e}^{2}$; the estimated persistence of $\mathrm{U}$ species at various air pressures is given in Figure 8. The persistence data are obtained at a distance $4 \mathrm{~mm}$ from the target surface. For the purpose of comparison, the persistence data for U I emission at various Ar ambient pressures are also given, taken from LaHaye et al. [13]. Comparing the persistence data obtained in air and Ar ambient environments, it can be seen that the emission persists at significantly longer times in an Ar ambient environment compared to air. The reduction in $\mathrm{U}$ line persistence in air could be caused by plasma quenching due to generation of oxides and other reactive species; however, further studies are needed. Rehse and co-workers [40] measured the decay constant, $\tau$, of $\mathrm{H}-\beta$ line emission from water plasma and noticed the emission lines decay much faster in air compared to Ar ambient. Their density estimate of the plasma also showed similar trends.

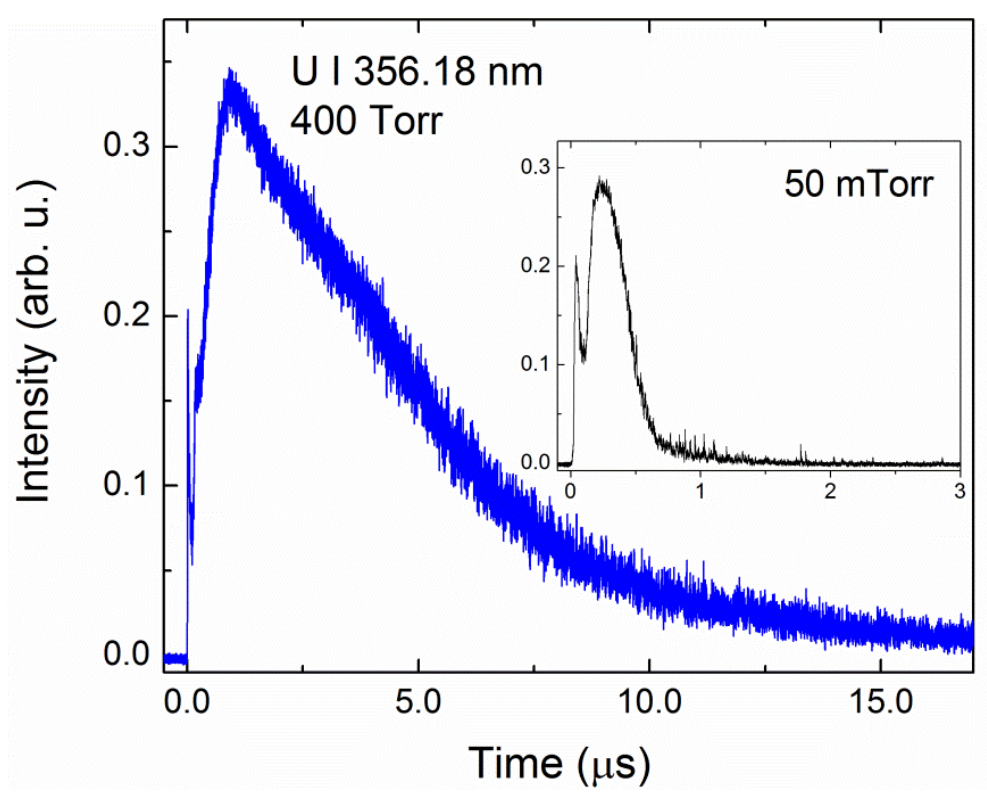

Fig. 7. The OTOF profile of U I at $356.18 \mathrm{~nm}$ recorded $4 \mathrm{~mm}$ from the target and at 400 Torr air pressure is given. The OTOF profile obtained at lower pressure condition (50 mTorr) is given in the inset. 


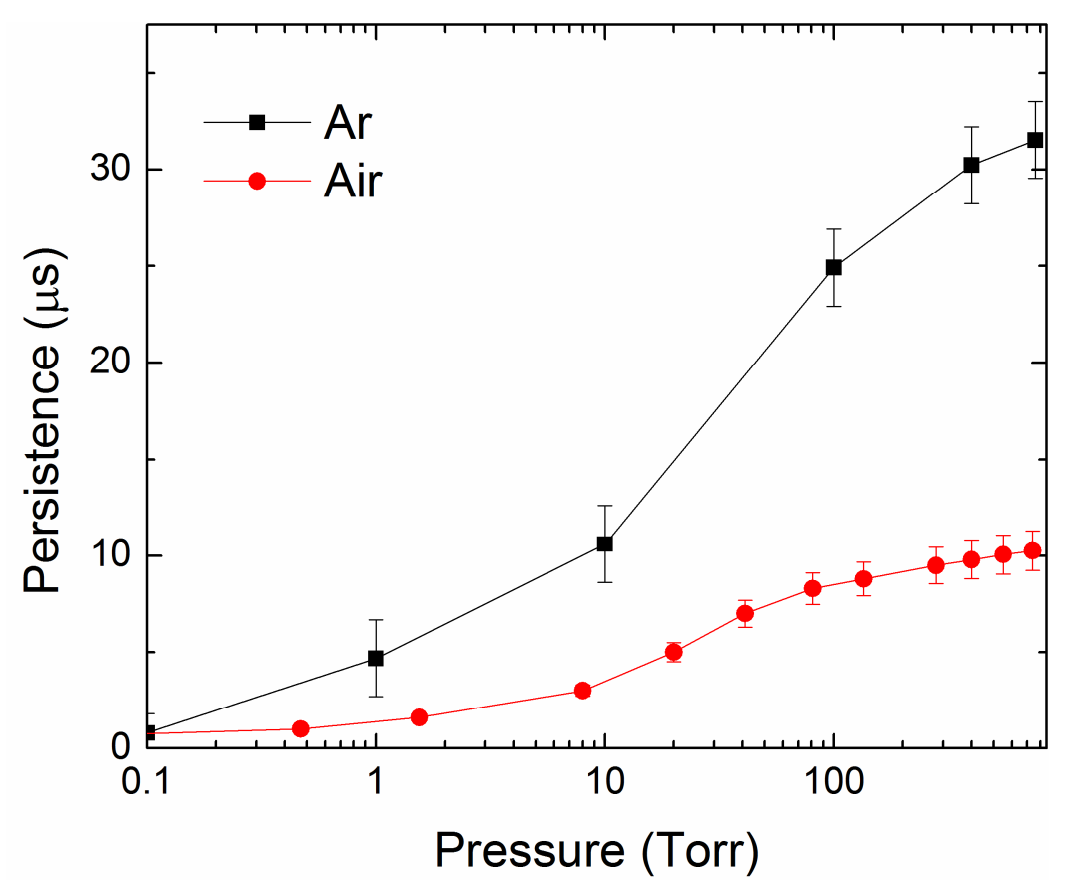

Fig. 8. The persistence of U I $356.18 \mathrm{~nm}$ emission at various ambient air pressure levels, taken $4 \mathrm{~mm}$ from the target surface, is given. The persistence data are obtained from OTOF profiles when the intensity drops to $1 / \mathrm{e}^{2}$. For comparison purpose, the persistence data for $\mathrm{U} I$ emission at various Ar ambient pressures are also given, taken from LaHaye et al.[13]

According to Eqn. (1), the $\mathrm{U}$ emission intensity is determined by both the $\mathrm{U}$ atomic number density and the excitation temperature, both of which have strong temporal and spatial variations. In vacuum conditions, the $\mathrm{U}$ undergoes a free expansion but the limited or negligible collisional excitation at later times results in a low excitation temperature, leading to emission with low persistence. As the pressure increases to moderate levels, the spatial extent of the plasma is reduced somewhat due to plasma confinement, but the increased excitation temperature [28] leads to an increased persistence of emission. For higher pressures, including atmospheric, the plasma confinement is such that the $\mathrm{U}$ emission is confined to regions close to the sample surface, as shown in Figure 6. However, the increased excitation temperature continues to increase the persistence of the $U$ emission. The results clearly demonstrate the value of space-time emission contours to study the evolution of the plasma properties, since the emission at any given point in space or time is insufficient to characterize the variation in all parameters. 


\section{Conclusions}

We investigated the spatio-temporal evolution of U-containing plasmas using optical emission spectroscopy under various air pressure levels ranging from vacuum to atmospheric conditions. The spectral emission features showed the presence of both excited neutral and ionic $U$ lines, and their intensities increased significantly with the addition of ambient air. Though ambient air pressure levels enhanced $\mathrm{U}$ line intensities, the improvement in $\mathrm{S} / \mathrm{B}$ ratio is found to be minimal due to enhanced background emission and due to changes in plasma chemistry [32]. The spatial dependence of S/B ratios showed that the analytical metrics of LA plumes change significantly with space and the optimal spatial positions vary with ambient pressure.

The estimated linewidths of the $\mathrm{U}$ emission were found to be less influenced by various broadening mechanisms initiated by increased pressure than Ca species in the multi-component plasma. The FWHM of $U$ line is found to increase by a factor of 2 when the pressure increased to atmospheric conditions, while in the same pressure range Ca I increased by a factor of 4 . Comparing the FWHMs of $\mathrm{U}$ I and Ca I at various pressure levels, it can be concluded that U lines appear to have lower Stark broadening, however, further study is needed to accurately determine the Stark broadening coefficient for the U I transition. Spatially and temporally resolved OTOF emission spectroscopy of excited uranium atoms demonstrated that the influence of ambient air pressure on the expansion hydrodynamics as well as the persistence of $U$ species in the plume. The presence of ambient air increased the temporal extension of U species. However, a comparison with $\mathrm{U}$ emission features in the presence of an inert gas showed the persistence of $U$ species in plasmas in ambient air is significantly reduced; this could be due to oxide and other reactive species formation; however, further studies are needed.

Acknowledgements: This work was supported by the DOE/NNSA Office of Nonproliferation and Verification Research and Development (NA-22). Pacific Northwest National Laboratory, a multiprogram national laboratory operated by Battelle for the U.S. Department of Energy under Contract DEAC05-76RL01830 


\section{FIGURE CAPTIONS}

Fig. 1. Typical emission spectrum of U-containing plasma in the range $350-370 \mathrm{~nm}$ is given. The major $\mathrm{U}$ emission lines are identified. The spectrum was recorded with a delay of $1 \mu \mathrm{s}$ after the onset of plasma formation and with an integration time of $5 \mu \mathrm{s}, 2 \mathrm{~mm}$ from the target surface with a pressure of 100 Torr.

Fig. 2. Intensity and $\mathrm{S} / \mathrm{B}$ ratio of $\mathrm{U}$ line emission at $356.18 \mathrm{~nm}$ is given with respect to air ambient pressure.

Fig. 3. Spatial dependence of S/B ratio of $U$ line emission at $356.18 \mathrm{~nm}$ is given for various ambient pressure levels.

Fig. 4. The estimated FWHMs of U I (356. $18 \mathrm{~nm})$ and Ca I $(422.67 \mathrm{~nm})$ lines are given with respect to various ambient pressure levels.

Fig. 5. The position-time contours obtained from U I $356.18 \mathrm{~nm}$ TOF are given for (a) 50mTorr, (b) 1 Torr and (c) 10 Torr air pressure levels. The scale bars given in the left figure correspond to signal levels measured using a PMT and intensity levels are normalized to similar scale levels for all contours.

Fig. 6. The position-time contours obtained from U I $356.18 \mathrm{~nm}$ TOF are given for (a) 100mTorr, and (b) 760 Torr air pressure levels. The scale bars given in the left figure correspond to signal levels measured using a PMT and intensity levels are normalized to similar scale levels for both contours.

Fig. 7. The OTOF profile of $U$ I at $356.18 \mathrm{~nm}$ recorded $4 \mathrm{~mm}$ from the target and at 400 Torr air pressure is given. The OTOF profile obtained at lower pressure (50 mTorr) condition is given in the inset.

Fig. 8. The persistence of U I $356.18 \mathrm{~nm}$ emission at various ambient air pressure levels, taken $4 \mathrm{~mm}$ from the target surface, is given. The persistence data is obtained from OTOF profiles when the intensity drops to $1 / \mathrm{e}^{2}$. For comparison purpose, the persistence data for U I emission at various Ar ambient pressures are also given, taken from LaHaye et al.[13] 


\section{REFERENCES}

[1] K. Mayer, M. Wallenius, I. Ray, Nuclear forensics - a methodology providing clues on the origin of illicitly trafficked nuclear materials, Analyst, 130 (2005) 433-441.

[2] N.S. Lloyd, R.R. Parrish, M.S.A. Horstwood, S.R.N. Chenery, Precise and accurate isotopic analysis of microscopic uranium-oxide grains using LA-MC-ICP-MS, J. Anal. Atom. Spectrom., 24 (2009) 752758.

[3] A.M. Duffin, K.W. Springer, J. Ward, K.D. Jarman, J.W. Robinson, C.E. Mackenzie, G.L. Hart, J.J. Gonzalez, D. Oropeza, R.E. Russo, D. Willingham, B.E. Naes, A. Fahey, G.C. Eiden, Femtosecond laser ablation multicollector ICPMS analysis of uranium isotopes in NIST glass, J. Anal. Atom. Spectrom., 30 (2015) 1100-1107.

[4] N.R. Taylor, M.C. Phillips, Differential laser absorption spectroscopy of uranium in an atmospheric pressure laser-induced plasma, Optics Letters, 39 (2014) 594-597.

[5] L.A. King, I.B. Gornushkin, D. Pappas, B.W. Smith, J.D. Winefordner, Rubidium isotope measurements in solid samples by laser ablation-laser atomic absorption spectroscopy, Spectrochim. Acta. Part B, 54 (1999) 1771-1781.

[6] G.C.Y. Chan, X.L. Mao, I. Choi, A. Sarkar, O.P. Lam, D.K. Shuh, R.E. Russo, Multiple emission line analysis for improved isotopic determination of uranium - a computer simulation study, Spectrochim. Acta. Part B, 89 (2013) 40-49.

[7] R.C. Chinni, D.A. Cremers, L.J. Radziemski, M. Bostian, C. Navarro-Northrup, Detection of Uranium Using Laser-Induced Breakdown Spectroscopy, Appl. Spectrosc., 63 (2009) 1238-1250.

[8] I. Choi, G.C.Y. Chan, X.L. Mao, D.L. Perry, R.E. Russo, Line Selection and Parameter Optimization for Trace Analysis of Uranium in Glass Matrices by Laser-Induced Breakdown Spectroscopy (LIBS), Appl. Spectrosc., 67 (2013) 1275-1284.

[9] D.A. Cremers, A. Beddingfield, R. Smithwick, R.C. Chinni, C.R. Jones, B. Beardsley, L. Karch, Monitoring Uranium, Hydrogen, and Lithium and Their Isotopes Using a Compact Laser-Induced Breakdown Spectroscopy (LIBS) Probe and High-Resolution Spectrometer, Appl. Spectrosc., 66 (2012) 250-261.

[10] F.R. Doucet, G. Lithgow, R. Kosierb, P. Bouchard, M. Sabsabi, Determination of isotope ratios using Laser-Induced Breakdown Spectroscopy in ambient air at atmospheric pressure for nuclear forensics, J. Anal. Atom. Spectrom., 26 (2011) 536-541.

[11] E.J. Judge, J.E. Barefield, J.M. Berg, S.M. Clegg, G.J. Havrilla, V.M. Montoya, L.A. Le, L.N. Lopez, Laser-induced breakdown spectroscopy measurements of uranium and thorium powders and uranium ore, Spectrochim. Acta. Part B, 83-84 (2013) 28-36.

[12] E.C. Jung, D.H. Lee, J.I. Yun, J.G. Kim, J.W. Yeon, K. Song, Quantitative determination of uranium and europium in glass matrix by laser-induced breakdown spectroscopy, Spectrochim. Acta. Part B, 66 (2011) 761-764.

[13] N.L. LaHaye, S.S. Harilal, P.K. Diwakar, A. Hassanein, Persistence of uranium emission in laserproduced plasmas, J. Appl. Phys., 115 (2014) 163301.

[14] M.Z. Martin, S. Allman, D.J. Brice, R.C. Martin, N.O. Andre, Exploring laser-induced breakdown spectroscopy for nuclear materials analysis and in-situ applications, Spectrochim. Acta. Part B, 74-75 (2012) 177-183. 
[15] A.I. Whitehouse, J. Young, C.P. Evans, Extreme LIBS, OSA Tecnical Digest, (2001) WA1-1. [16] A.I. Whitehouse, J. Young, I.M. Botheroyd, S. Lawson, C.P. Evans, J. Wright, Remote material analysis of nuclear power station steam generator tubes by laser-induced breakdown spectroscopy, Spectrochim. Acta. Part B, 56 (2001) 821-830.

[17] J.R. Wachter, D.A. Cremers, Determination of Uranium in Solution Using Laser-Induced Breakdown Spectroscopy, Appl. Spectrosc., 41 (1987) 1042-1048.

[18] A. Sarkar, D. Alamelu, S.K. Aggarwal, Determination of thorium and uranium in solution by laserinduced breakdown spectrometry, Appl. Opt., 47 (2008) G58-G64.

[19] A. Sarkar, D. Alamelu, S.K. Aggarwal, Determination of trace constituents in thoria by laser induced breakdown spectrometry, J. Nucl. Mater., 384 (2009) 158-162.

[20] W. Pietsch, A. Petit, A. Briand, Isotope ratio determination of uranium by optical emission spectroscopy on a laser-produced plasma - basic investigations and analytical results, Spectrochim. Acta. Part B, 53 (1998) 751-761.

[21] P.L. Smith, C. Heise, J.R. Esmond, R.L. Kurucz, in: Atomic Spectral line database (http://www.pmp.uni-hannover.de/cgi-bin/ssi/test/kurucz/sekur.html), accessed 09/26/2014.

[22] J. Blaise, L.J. Radziemski, Energy-Levels of Neutral Atomic Uranium (Ui), J. Opt. Soc. Am., 66 (1976) 644-659.

[23] J. Blaise, J.F. Wyart, J. Verges, R. Engleman, B.A. Palmer, L.J. Radziemski, Energy-Levels and Isotope Shifts for Singly Ionized Uranium (U-Ii), J. Opt. Soc. Am. B, 11 (1994) 1897-1929.

[24] C.H. Corliss, Line Strengths and Lifetimes of Levels in Neutral Uranium, J Res Nbs a Phys Ch, 80 (1976) 1-7.

[25] C.H. Corliss, Oscillator-Strengths for Lines of Ionized Uranium (Uii), J Res Nbs a Phys Ch, 80 (1976) 429-438.

[26] S.S. Harilal, C.V. Bindhu, M.S. Tillack, F. Najmabadi, A.C. Gaeris, Internal structure and expansion dynamics of laser ablation plumes into ambient gases, J. Appl. Phys., 93 (2003) 2380-2388.

[27] H.R. Griem, Principles of Plasma Spectroscopy, Cambridge University Press, Cambridge, 1997. [28] N. Farid, S.S. Harilal, H. Ding, A. Hassanein, The emission features and expansion dynamics of nanosecond laser ablation plumes under varying ambient pressures J. Appl. Phys., 115 (2014) 033107.

[29] J.R. Freeman, P.K. Diwakar, S.S. Harilal, A. Hassanein, Improvements in discrimination of bulk and trace elements in long-wavelength double-pulse LIBS, Spectrochimica Acta B, 102 (2014) 36-41. [30] B.T. Fisher, H.A. Johnsen, S.G. Buckley, D.W. Hahn, Temporal gating for the optimization of laserinduced breakdown spectroscopy detection and analysis of toxic metals, Appl. Spectrosc., 55 (2001) 1312-1319.

[31] S.V. Shabanov, I.B. Gornushkin, Two-dimensional axisymmetric models of laser induced plasmas relevant to laser induced breakdown spectroscopy, Spectrochim. Acta. Part B, 100 (2014) 147-172. [32] R. Saad, D. L'Hermite, B. Bousquet, Unexpected temporal evolution of atomic spectral lines of aluminum in a laser induced breakdown spectroscopy experiment, Spectrochim. Acta. Part B, 101 (2014) 330-334.

[33] P.K. Diwakar, S.S. Harilal, A. Hassanein, M.C. Phillips, Expansion dynamics of ultrafast laser produced plasmas in the presence of ambient argon, J. Appl. Phys., 116 (2014) 133301.

[34] S.S. Harilal, B. O'Shay, M.S. Tillack, M.V. Mathew, Spectroscopic characterization of laser-induced tin plasma, Journal of Applied Physics, 98 (2005) 013306. 
[35] S.S. Harilal, T. Sizyuk, A. Hassanein, D. Campos, P. Hough, V. Sizyuk, The effect of excitation wavelength on dynamics of laser-produced tin plasma, Journal of Applied Physics, 109 (2011) 063306. [36] R.K. Singh, O.W. Holland, J. Narayan, Theoretical-Model for Deposition of Superconducting ThinFilms Using Pulsed Laser Evaporation Technique, J. Appl. Phys., 68 (1990) 233-247.

[37] N. Smijesh, R. Philip, Emission dynamics of an expanding ultrafast-laser produced Zn plasma under different ambient pressures, J. Appl. Phys., 114 (2013) 093301.

[38] K.F. Al-Shboul, S.S. Harilal, A. Hassanein, Gas dynamic effects on formation of carbon dimers in laser-produced plasmas, Appl. Phys. Lett., 99 (2011) 131506.

[39] S.S. Harilal, B. O'Shay, Y.Z. Tao, M.S. Tillack, Ambient gas effects on the dynamics of laserproduced tin plume expansion, Journal of Applied Physics, 99 (2006) 083303.

[40] M. Adamson, A. Padmanabhan, G.J. Godfrey, S.J. Rehse, Laser-induced breakdown spectroscopy at a water/gas interface: A study of bath gas-dependent molecular species, Spectrochim. Acta. Part B, 62 (2007) 1348-1360. 